\title{
Shape control in concave metal nanoparticles by etching $\dagger$
}

\author{
Qiang Li, (D) a Marcos Rellán-Piñeiro, (iD a Neyvis Almora-Barrios, (D) a \\ Miquel Garcia-Ratés, (ID a loannis N. Remediakis (DD b and Núria López (D) *a
}

\begin{abstract}
The shape control of nanoparticles constitutes one of the main challenges in today's nanotechnology. The synthetic procedures are based on trial-and-error methods and are difficult to rationalize as many ingredients are typically used. For instance, concave nanoparticles exhibiting high-index facets can be obtained from Pt with different $\mathrm{HCl}$ treatments. These structures present exceptional capacities when are employed as catalysts in electrochemical processes, as they maximize the activity per mass unit of the expensive material. Here we show how atomistic simulations based on density functional theory that take into account the environment can predict the morphology for the nanostructures and how it is even possible to address the appearance of concave structures. To describe the control by etching, we have reformulated the Wulff construction through the use of a geometric model that leads to concave polyhedra, which have a larger surface-to-volume ratio compared to that for nanocubes. Such an increase makes these sorts of nanoparticles excellent candidates to improve electrocatalytic performance.
\end{abstract}

\section{Introduction}

The properties of noble metal nanoparticles depend on their morphology and surface crystalline orientation and, thus, the specific control of the architectures is one of the major challenges in nanotechnology. ${ }^{1-7}$ Shape control has been achieved and several synthetic methods allow the production of selected polyhedra limited by convex surfaces (cubes, octahedra, tetrahedra, and rods). ${ }^{8-10}$ Many of the synthetic processes are based on the generation of a seed by a fast reduction of a metal salt, which is then grown on a solution containing halides and/or surfactants with a milder reductive agent. Recent studies have shown the efficiency of biomolecules in recognizing specific crystallographic facets and affecting nanocrystal shapes. ${ }^{11,12}$ The mechanism of shape control has been proposed to be kinetic or thermodynamic and is a consequence of the solution ingredients and conditions. ${ }^{13}$ In recent years, there has been considerable interest in the synthesis of concave nanoparticles (see Fig. 1). ${ }^{2,14}$ These structures have unique potential in electrocatalysis because of their exposed

${ }^{a}$ Institute of Chemical Research of Catalonia, ICIQ, The Barcelona Institute of Science and Technology, Av. Països Catalans, 16, 43007 Tarragona, Spain. E-mail: nlopez@iciq.es

${ }^{b}$ Department of Materials Science and Technology, University of Crete, and Institute of Electronic Structure and Laser, FORTH, Heraklion, 71003, Greece $\dagger$ Electronic supplementary information (ESI) available: Calculated surface and binding energies, resulting Wulff structures, and details on the ab initio thermodynamics formalism and on the geometric model. See DOI: 10.1039/c7nr03889e

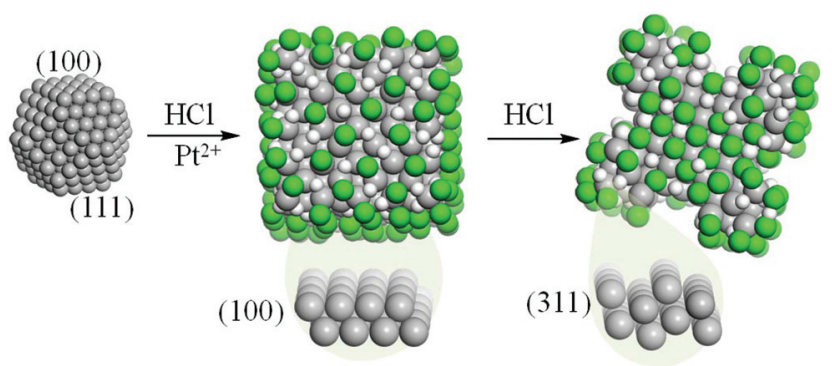

Fig. 1 Atomic representation of the structures formed during the synthetic process of Pt nanoparticles based on the fast reduction of a metal salt that grows in a solution with a different amount of $\mathrm{HCl}$. The insets highlight the most stable surfaces in each stage of the process. The scheme is adapted from ref. 2. Color code for the surface atoms: Pt in grey, $\mathrm{Cl}$ in green, and $\mathrm{H}$ in white.

high-index facets ${ }^{5,15}$ that encompass a larger surface area compared to that in convex systems and result in more active sites. ${ }^{14-19}$ In wet chemistry, two strategies have been proposed to generate concave nanocrystals of active metals: (i) directionally controlled overgrowth ${ }^{20,21}$ and (ii) site-specific dissolution. ${ }^{22}$ Overgrowth relies on the fact that capping agents, bound to specific facets, modify the relative growth rates leading to concave polyhedra. This is the case of Pt octapods exhibiting the $\{411\}$ facets, or $\mathrm{Ag}$ nanostructures that are formed when amines or $\mathrm{Cu}^{2+}$ ions are added. ${ }^{15,23}$ Octapods were also indicated to appear by kinetically controlled overgrowth in the case of $\mathrm{Rh}$ with bromide as a capping agent. ${ }^{24}$ 
Alternatively, concave nanoshapes can be formed by partial dissolution of a larger nanoparticle. The region that experiences the faster dissolution evolves into a concave surface. Therefore, the strength of the etchants needs to be finely tuned in order to obtain the desired shape. ${ }^{25}$

Halides and hydrogen halides form dense phases on metal surfaces. ${ }^{26}$ For $\mathrm{Au}$ and $\mathrm{Ag}$, halides are employed as structure directing coatings that show a large preference for the $\{100\}$ planes, and thus for cubes. ${ }^{27}$ This has been recently confirmed computationally, showing a thermodynamic control of the nanoparticle shape. ${ }^{28}$ Experiments on more reactive metals, like Pt or Pd, have found that hydrogen halides at certain concentrations lead to the formation of concave nanoparticles. ${ }^{2,3,29}$ In these cases, the process of the formation of high-index facets requires available low index-planes like $\{100\}$ and $\{111\}$ surfaces that serve as templates. ${ }^{14}$

The morphology of nanoparticles can be explored through the systematic use of theoretical tools based on Density Functional Theory, DFT, coupled with the Wulff construction, either in the continuum ${ }^{30,31}$ or atomistic versions, ${ }^{32,33}$ or by creating the environment through first-principles thermodynamics. $^{34}$ In the experiments, concave nanoparticles are produced in an acid/watery environment, in open air and with the $\mathrm{Pt}$ precursor in the solution. The full theoretical study would require addressing all the stages from nucleation to growth and including all the reactive ingredients which make the prediction of the shape computationally unaffordable. Alternatively, we have shown previously ${ }^{28,35}$ that a much better understanding of the shape controlling factors can be obtained by addressing the problem of the nanoparticle shape through first principles and by sequentially increasing the level of complexity (i.e. with the presence of the solvent, additives). The lack of a methodology equivalent to the Wulff construction for concave objects poses an additional challenge. In this study, we aim at describing the formation of cubic Pt nanoparticles and how they lead to concave structures when $\mathrm{HCl}$ concentration increases and at describing an alternative to the Wulff construction for concave structures.

\section{Methods}

We have performed DFT calculations with the Vienna $a b$ initio simulation package code (VASP version 5.3.3) (6,37 $^{36}$ with the Perdew-Burke-Ernzerhof (PBE) exchange-correlation functional. $^{38}$ Inner electrons were represented by projector augmented wave (PAW) potentials ${ }^{39,40}$ with a kinetic cutoff energy of $400 \mathrm{eV}$, and dispersion contributions with D2 parameters complemented with our reparametrized values for metals. ${ }^{41,42}$ The corresponding $k$-point samplings were denser than $0.3 \AA^{-1}$. We considered several low-index planes, (100), (111), and (110), and also the open surfaces that might be present in particle-etched concave structures, that is, (211), (311), (411) and (511) planes which contain the $\{111\}$ steps and the $\{100\}$ terraces. The models for all the Pt surfaces were constructed with 5-9 layers depending on the degree of openness of the surfaces to ensure convergence in all cases. The topmost 2-4 layers were relaxed during optimization, while the remaining layers were kept fixed to mimic the bulk. The structures were interleaved under $12 \AA$ vacuum. $\mathrm{HCl}$ was adsorbed only on the topmost surface and the dipole correction was employed to correct potential spurious terms arising from the asymmetry of the slabs. ${ }^{43}$ We used $a b$ initio atomistic thermodynamics to understand the effect of the concentration of different adsorbed species on the stability and growth of Pt nanoparticle surfaces. $^{34}$ Solvent contributions were added via the VASP-MGCM (VASP-Multigrid Continuum Model). ${ }^{44}$ To illustrate how theoretical simulations can account for the etching of Pt nanoparticles, we have generated a finite cubic model of $16 \AA$ A side, representing the most likely growth morphology. The surface was completely covered with $\mathrm{HCl}$ and the total stoichiometry was $365 \mathrm{Pt}$ atoms and $148 \mathrm{HCl}$ units in the form of a monolayer of a rock-salt-like structure (see Fig. S2 $\dagger$ ). The so-built structure was then allowed to relax and during this step some $\mathrm{HCl}$ molecules were formed and left the surface due to the repulsion between them. These molecules were then removed from the simulation box leaving the final Pt-cube structure surrounded by $119 \mathrm{HCl}$ units. Pitting was then tested at different positions of the naked $\mathrm{Pt}_{365}$ and $\mathrm{Pt}_{365}(\mathrm{HCl})_{119}$ : in the middle of a facet, in the edge, and at the corner positions. Finally, we computed the change in energy due to the addition of an extra Pt atom to the $\mathrm{Pt}_{365}$ and $\mathrm{Pt}_{365}(\mathrm{HCl})_{119}$ at the face positions. A data-set collection of computational results is available in the ioChem-BD repository. ${ }^{45}$

\section{Results and discussion}

Experiments have evidenced that Pt nanoparticles present concave faces upon exposure to high concentrations of $\mathrm{HCl}^{2}$ Simulations have been carried out to explain these observations. The present simulations might seem simple but we would like to point out the following: (i) this is the first ever approach that involves the use of density functional theory to explore the appearance of concave nanoparticles by etching. (ii) Solvent contributions are employed when needed through a continuum model. Although this is not the best situation, because explicit water molecules could have a role, it simplifies the chemical approach whilst avoiding a discussion based only on gas-phase energies. (iii) The contribution of oxidants $\left(\mathrm{O}_{2}\right)$ that might be present in the solution has not been considered. Again, a proper representation of the full phenomena of shape control would be needed to integrate the mechanistic contribution of these species. (iv) However, even if the models presented here are rather simple, they do allow the formulation of a concave version of the Wulff construction. The Wulff construction has served to understand the equilibrium structure of nanoparticles since 1901. To our knowledge, this is the first attempt to predict the theoretically concave shapes of nanoparticles in a generalized framework.

The first point that is addressed in the simulations is the estimation of the adsorption energy of $\mathrm{HCl}$ molecules on all 


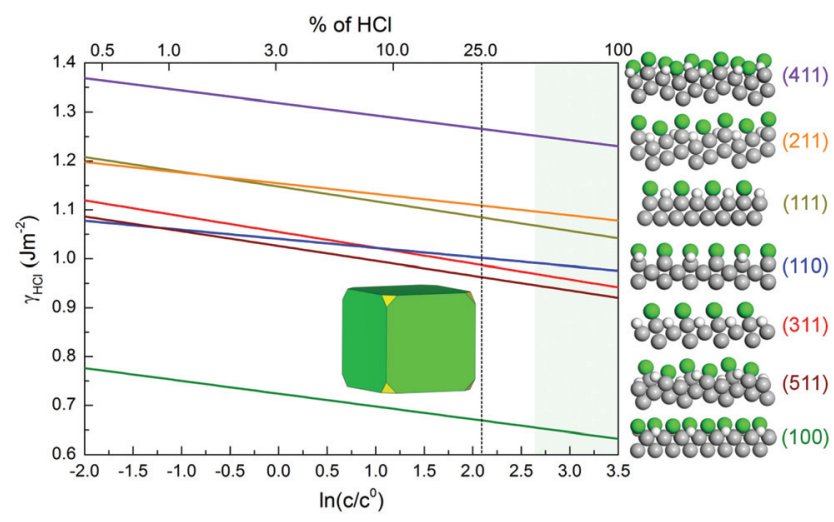

Fig. 2 (Left) Surface energy for different planes as a function of the $\mathrm{HCl}$ concentration $\left(c^{0}=1 \mathrm{M}\right)$ at room temperature and (right) side view of the different surfaces with the densest $\mathrm{HCl}$ coverage. The inset represents the nanoparticle morphology, as derived from the Wulff construction at $25 \%$ of $\mathrm{HCl}$ (dashed line). The shaded area corresponds to $\mathrm{HCl}$ concentrations where pitting occurs, as calculated from our kinetic model. Color code for the Pt surfaces: (100) green, (110) blue, (111) olive, (211) orange, (311) red, (411) purple and (511) wine. Atomic color code as in Fig. 1.

relevant Pt surfaces. These quantities are needed to predict the equilibrium shape of the nanoparticle in an interacting environment using the Wulff construction method. ${ }^{30}$ The interaction of $\mathrm{HCl}$ on these surfaces with the densest coverage was calculated with respect to the solvated molecules. The results for adsorption energies and atom-atom distances are summarized in section S2. $\dagger$ Fig. 2 (right) shows the side view of the densest $\mathrm{HCl}$ packing on low-index facets, such as (100), (110), and (111), and also on stepped surfaces (211), (311), (411) and (511). The HCl molecules have been found to adsorb preferentially on the (100) Pt surfaces, resulting in a large value for the binding energy per $\mathrm{HCl}$ molecule $(-1.11 \mathrm{eV}$ per $\mathrm{HCl})$. Note that the adsorption of $\mathrm{NaCl}$ also considered in ref. 2 is almost thermoneutral, so the nanoparticle shape control would only be induced by $\mathrm{Cl}^{-}$adsorption as in the case of $\mathrm{Ag} @ \mathrm{Au}^{28}$ (therefore, we do not discuss it further). In this surface, the $\mathrm{Cl}$ and $\mathrm{H}$ atoms are placed on the bridge sites of the Pt surface at a distance of $1.934 \AA$ And $1.011 \AA$ from the surface, respectively. Surface energies calculated with DFT methods are known to deviate from experimental data. ${ }^{46}$ However, DFT-based results for binding energies are not affected in the same way. ${ }^{42}$

To predict the shape of the nanoparticles in the HCl-water environment, we have adopted an $a b$ initio thermodynamics framework, ${ }^{34}$ see section $\mathrm{S} 4, \uparrow$ that allows the estimation of the surface energy for each of the surfaces as a function of the acid concentration, $c$. For this purpose, we considered the chemical potential of the species as the reference in solution at room temperature. The results for the surface energy for the HClcovered Pt surfaces, $\gamma_{\mathrm{HCl}}$, at different $\mathrm{HCl}$ concentrations are shown in Fig. 2 (left). The range of $\mathrm{HCl}$ concentrations corresponds to values between $0.5 \%$ and $100 \%$ (see Table $S 5 \dagger$ ).
The $a b$ initio thermodynamics indicates that the preferred configurations are dense phases with high concentrations of adsorbed $\mathrm{HCl}$. The general trend shown in Fig. 2 is that the (100) surface is the most stable over the whole range of concentrations. With regard to the remaining surfaces, it is important to note that, although the (311) and (511) faces are less stable than the (110) surface at low $\mathrm{HCl}$ concentrations, the situation is the opposite for large $\mathrm{HCl}$ amounts. In order to validate our approach, we repeated the calculations for the (100) surface considering the system solvated by the continuum model in water. The largest deviation in the calculated $\gamma_{\mathrm{HCl}}$ with respect to the same quantity for the systems in vacuum is lower than $1 \%$. Therefore, unsolvated surface energies were employed and solvation was considered only for the reference $\mathrm{HCl}$ molecule (section S2 $\dagger$ ), in the definition of the binding energy. The inset in Fig. 2 shows that the application of the Wulff construction at $25 \%$ of $\mathrm{HCl}$ concentration results in a nanocube (see section $\mathrm{S} 3 \dagger$ ).

Increasing the acid concentration led to the erosion of the cubes. Experiments showed that at a $\mathrm{HCl}$ concentration of $37 \%{ }^{2}$ the corresponding structure obtained from our Wulff construction is no longer valid. Increasing the $\mathrm{HCl}$ concentration further causes the formation of bone-like structures without increasing the nanoparticle size. ${ }^{2}$ This is due to the effect of the acid. As the deposition rate starts to become lower than the etching one, and therefore pitting starts. ${ }^{3}$ In order to understand how pitting occurs, we calculated the reaction energies for dissolution at relevant acid concentrations. We started by studying this dissolution process on the thermodynamically preferred surface: (100), and on a high-index plane: the (311) facet. The removal of a Pt atom from the (100) surface is $0.21 \mathrm{eV}$ easier than that from the (311) one. This energy difference relies on the different $\mathrm{HCl}$ configuration that we have on both the surfaces. In the case of the (100) facet, $\mathrm{H}$ and $\mathrm{Cl}$ atoms are placed at the bridge positions between $\mathrm{Pt}$ atoms, whereas for the (311) surface, $\mathrm{H}$ and $\mathrm{Cl}$ atoms are placed on top of the Pt atoms (see Fig. 3a and b). When a Pt atom is removed from both the surfaces, there is no change in the configuration of the $\mathrm{HCl}$ network in the (100) facet, whereas for the (311) facet, a $\mathrm{Cl}$ atom occupies the hollow left by Pt. Thus, although for this last surface the $\mathrm{Cl}$ atom approaches a Pt atom, the $\mathrm{HCl}$ network is distorted locally and leads to a less favorable situation in terms of energy compared to the pitting in the (100) facet.

Similarly, for the $\mathrm{Pt}_{365}$ nanoparticle, we calculated the energy gain to depositing an isolated Pt atom together with the energy cost to remove a Pt atom from the corner, step and the (100) facet. In the case of naked Pt nanoparticles, the easiest Pt removal corresponds to the lowest atom coordination, the corners being $1.75 \mathrm{eV}$ more thermodynamically easy to be removed than the atoms at the face centers (see Table S6 $\dagger$ ). The situation changes for HCl-covered nanoparticles (see Fig. 3c and d). In this case, the pitting at the edge and facet positions is almost equivalent and the corresponding configurations are $0.94 \mathrm{eV}$ more stable than removing one atom at the corner, thus the stability goes as: corner $>$ edge 
(100)

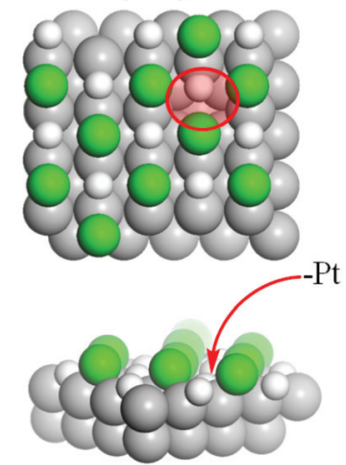

c)

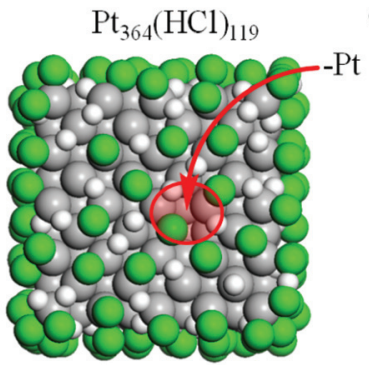

b)
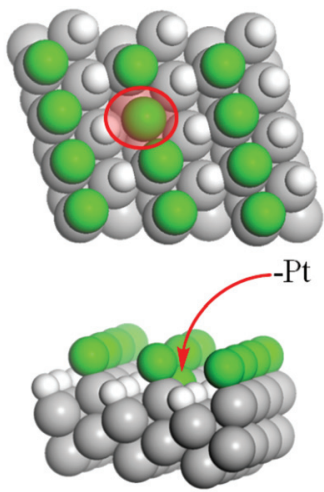

d)

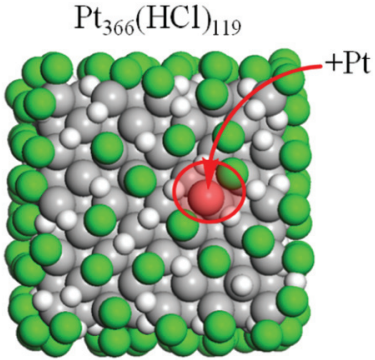

Fig. 3 Optimized structures after a $\mathrm{Pt}$ atom removal of $\mathrm{HCl}$ on (a) the $\mathrm{Pt}(100)$, (b) $\mathrm{Pt}(311)$ surfaces, as well as on (c) the $\mathrm{Pt}_{365}(\mathrm{HCl})_{119}$ nanocube, and (d) after a Pt atom addition in the later system. Atomic color code as in Fig. 1. The positions of the removed and added Pt atoms are highlighted with a red circle.

$\approx$ facet. However, for a cube with side length $a$ and $N$ atoms side, the number of edge atoms $N_{\mathrm{e}}$ is linear with $N$, while the number of face atoms $N_{\mathrm{f}}$ is quadratic. Therefore, $N_{\mathrm{f}}$ is one order of magnitude larger than $N_{\mathrm{e}}$ and, thus, the pitting shall start readily at the facets.

Although the presence of oxidative agents and explicit water models would be needed to have a complete picture of the synthesis and shape control of the nanoparticles, the computed results have already provided some hints on these phenomena.

With the calculated values, we propose a kinetic model to identify at which $\mathrm{HCl}$ concentration the pitting starts. The energy references have been taken as soluble $\mathrm{H}_{2} \mathrm{PtCl}_{6}$ units. This model relies on two equations:

Deposition $\mathrm{Pt}_{365}+\mathrm{H}_{2} \mathrm{PtCl}_{6}+4 \mathrm{H}_{3} \mathrm{O}^{+} \rightarrow \mathrm{Pt}_{366}+6 \mathrm{HCl}+4 \mathrm{H}_{2} \mathrm{O}$

$$
\text { Elimination } \mathrm{Pt}_{365}+6 \mathrm{HCl} \rightarrow \mathrm{Pt}_{364}+\mathrm{H}_{2} \mathrm{PtCl}_{6}+4 \mathrm{H}^{+}
$$

The kinetic coefficients ${ }^{47}$ for deposition and elimination can be traced back to the values for deposition $(-4.63 \mathrm{eV}$ at the facet of the HCl-decorated $\left.\mathrm{Pt}_{365}\right)$ and elimination (4.17 eV from the facet of the HCl-decorated $\left.\mathrm{Pt}_{365}\right)$. For simplicity, we consider the kinetics of the above equations to be governed by power-low rates. Solvent effects are only considered through the continuum model. Although an atomistic insight ${ }^{48}$ would be relevant to unravel the specific effect of the solvent, this point is beyond the aim of the present work. At a given value for the $\mathrm{HCl}$ concentration, when pitting starts, the deposition and elimination rates shall be equal and we can write the following equation

$$
k_{\mathrm{dep}}\left[\mathrm{Pt}_{365}\right]\left[\mathrm{H}_{2} \mathrm{PtCl}_{6}\right]\left[\mathrm{H}_{3} \mathrm{O}^{+}\right]^{4}=k_{\mathrm{el}}\left[\mathrm{Pt}_{365}\right][\mathrm{HCl}]^{6}
$$

Considering the experimental conditions: room temperature and the concentration of $\mathrm{Pt}$ in the solution, $\left[\mathrm{H}_{2} \mathrm{PtCl}_{6}\right]=$ $0.1 \mathrm{M}$ and $\mathrm{pH}=0$, the $[\mathrm{HCl}]$ concentration at which the pitting starts is $43 \% \mathrm{HCl}$. Experimentally, the transition between Pt nanocubes and bonelike structures is observed at $37 \% .^{2}$ Given the simplicity of our model, the agreement between the two thresholds for the pitting is remarkable. In addition, our results qualitatively agree with the increase in the (100) surface area of up to $25 \% \mathrm{HCl}$ ( $43 \%$ in our case) and with its decrease for higher concentrations. From that point onwards, the surface area of surfaces like the 311 and 511 (which involve a higher number of steps and defects) increases. ${ }^{2}$

As the Wulff theorem always results in convex objects, we devised a new procedure to consider etched nanostructures obtained: (i) its final shape, (ii) surface(s) exposed, and (iii) the surface-to-volume ratio. Starting from the nanocube limited $\{100\}$ planes and given that the pitting starts at the center of the facet, the formation of the convex structure can be found as a volume excavation from this pitting point. ${ }^{15}$ Therefore, the process of forming a high-index faceted concave nanoparticle consists of the removal of an object (in our case, a tetragonal pyramid), made of the $\{h l l\}$-like planes from each face of a nanocube $(h, k$, and $l$ are Miller indices with $k=l$ and $h>k$, see Fig. 4a). Varying the concentration of $\mathrm{HCl}$ would then correspond to a different degree of intersection between solids. In the beginning, the facet excavated structures resemble the bonelike ones reported in ref. 2. In the limit where the $\{100\}$ planes are completely excavated out, the resulting structure is an octapod containing, in our case, only the $\{311\}$ planes (Fig. 4b). As mentioned above, it is important to point out that a concave nanoparticle containing just the $\{311\}$ planes can only be obtained if the pitting occurs at the center of the $\{100\}$ faces. If it occurred either at the corners or at the edge centers, the resulting structures would always contain the $\{100\}$ and $\{311\}$ planes simultaneously, and would never have sharp corners. A full derivation of the nanostructure formation, bonelike to octapods in this case, is presented in section S6. $\dagger$

The final effect of the degree of penetration of the etching volume (red in Fig. 4) is represented by the $\delta$ parameter, which is the shortest distance between the corners of the pyramidal pit and the edges of the cubic particle (see Fig. $4 \mathrm{~b}$ ). The parameter $\delta$ depends on the concentration $c$ of $\mathrm{HCl}$ (see section S6.2†). Therefore, for low etching values, the surface area of the nanoparticle does not change much (Fig. 4c). At intermediate values, the composition of the surface is $50 \%$ that of the $\{100\}$ and $\{311\}$ stepped surfaces, and only when the octapods appear, the total surface area decreases by about $45 \%$ of the initial value, being mostly of the $\{311\}$ type. The effect on the volume is more pronounced. Although at $\delta=0$ the volume is 

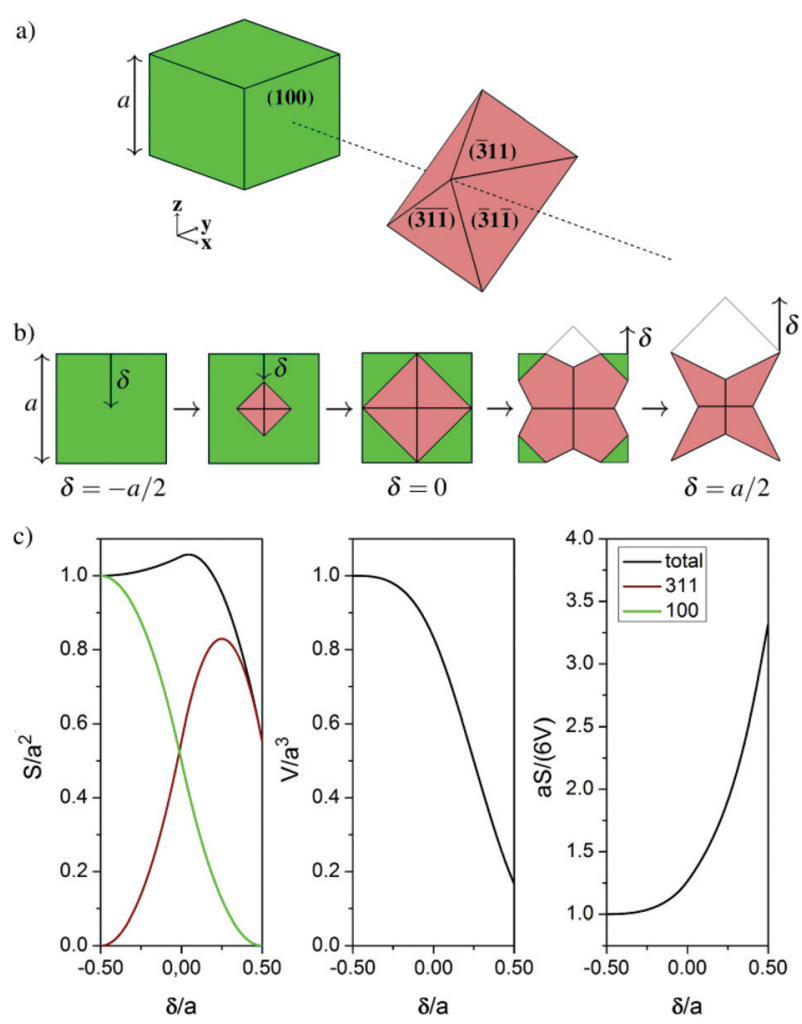

Fig. 4 (a) The formation of etched nanoparticles exhibiting the $\{311$ planes can be described as a process where a tetragonal pyramid composed of the $\{311\}$ planes intersects a cube at each face center. (b) Resulting structures for different $\mathrm{HCl}$ concentrations in the $y-z$ plane (concentration increases from left to right). The gray line corresponds to the intersection between the tetragonal pyramid with the plane $x=a / 2$. The parameter $\delta$ (see section $\mathrm{S} 6 \dagger$ ), which is shown for some of the cases, is measured from the edge of each face. (c) Surface area (left), volume (middle) and surface-to-volume ratio (right) for the nanoparticles as a function of $\delta$. All quantities are scaled by the respective values for a cube with side length $a$. The color code for the surfaces is the same as shown in Fig. 2.

about $80 \%$, when the octapod is formed the volume is only around $20 \%$ that of the nanocube. The most striking characteristic of these nanoobjects is the increase in the surface-tovolume ratio upon etching. At intermediate values of $\delta$, the effect is not pronounced (marginal 10\% increase), but it exponentially grows for $\delta / a$ larger than 0.25 . Thus, after this severe etching (represented by the fourth structure in Fig. 4b), the surface-to-volume ratio is doubled and, under the most favorable conditions, it reaches a 3-4-fold increase. Such atom economy is essential to improve electrocatalytic performance.

To sum up, the acid control on the shape of the nanoparticle works as follows. Firstly, the nanostructures grow exhibiting the $\{100\}$ surfaces as predicted from ab initio thermodynamics and reinforced by the Wulff construction. Once the chemical potential of the acid in the solution is too high, the deposition and consequent growth are no longer preferred over elimination and etching. This can be inferred by considering the chemical potential in the corresponding microkinetic equations. Here, the starting pitting point is important as it determines the final nanoparticle shape. A detailed balance between the number of potential pitting points - corners, edges, and facets - and the thermodynamic demand for this particular elimination is required to obtain the etched shape. Once the starting pitting point, in our case the face center, has been determined, the final equilibrium structure can be obtained by a geometrical model involving the intersection of our nanoobject with a polyhedron composed of the next stable surface at the pitting point. The impact on the geometric parameters can then be described through a model which, in our particular case, results in a surface-to-volume enhancement of up to 3-4-fold which improves the atom economy in these types of materials.

\section{Conclusions}

Theoretical calculations hold the key for an atomistic understanding of the control in the synthesis of nanostructures even when concave structures are generated. We have presented a formulation that describes the formation of concave Pt nanoparticles in the HCl-water environment. Our results based on ab initio thermodynamics predict cubic nanoparticles for any $\mathrm{HCl}$ concentration. However, at a certain $\mathrm{HCl}$ concentration, the gain for adding an extra Pt atom to the nanoparticle equilibrates with that of etching. Therefore, pitting starts at the most favorable elimination position, the facets. From this point, a geometric model based on the intersection of a nanocube and a polyhedron made of the $\{h l l\}$ planes predicts the equilibrium nanoparticle shape as a function of the degree of intersection, $\delta$, between both the figures. When this parameter, which can be correlated with $\mathrm{HCl}$ concentration, adopts its maximum value, the structures are the octapods but resemble bonelike for smaller values as in the experiments. For the limiting case of octapods made of the $\{311\}$ planes, the volume decreases $80 \%$ with respect to that for the cubic nanoparticle and the surface-to-volume ratio reaches a 3-4-fold increase. The resulting insights advance the understanding of the role of additives during metal nanoparticle growth and etching and can be extended to other metals and structures, thus opening a new perspective in understanding the control of new shapes and architectures.

\section{Conflicts of interest}

There are no conflicts of interest to declare.

\section{Acknowledgements}

The authors acknowledge the MINECO (project CTQ201568770-R and grants FPDI-2013-16194, SEV-2013-0319, and SEVP-2014-068237) for financial support. We are grateful to Professors J. M. Feliu and L. M. Liz-Marzan, for useful discussions. 


\section{References}

1 E. C. Dreaden, A. M. Alkilany, X. H. Huang, C. J. Murphy and M. A. El-Sayed, Chem. Soc. Rev., 2012, 41, 27402779.

2 R. A. Martínez-Rodríguez, F. J. Vidal-Iglesias, J. SollaGullón, C. R. Cabrera and J. M. Feliu, J. Am. Chem. Soc., 2014, 136, 1280-1283.

3 T. Yu, D. Y. Kim, H. Zhang and Y. Xia, Angew. Chem., Int. Ed., 2011, 50, 2773-2777.

4 X. Xie, G. Gao, Z. Pan, T. Wang, X. Meng and L. Cai, Sci. Rep., 2015, 5, 8515.

5 H. Mistry, A. S. Varela, S. Kühl, P. Strasser and B. R. Cuenya, Nat. Rev. Mater., 2016, 1, 16009.

6 H. Heinz, C. Pramanik, O. Heinz, Y. Ding, R. K. Mishra, D. Marchon, R. J. Flatt, I. Estrela-Lopis, J. Llop, S. Moya and R. F. Ziolo, Surf. Sci. Rep., 2017, 72, 1-58.

7 Y. Xia and X. Yang, Acc. Chem. Res., 2017, 50, 450-454.

8 C. Burda, X. B. Chen, R. Narayanan and M. A. El-Sayed, Chem. Rev., 2005, 105, 1025-1102.

9 M. Grzelczak, J. Pérez-Juste, P. Mulvaney and L. M. LizMarzán, Chem. Soc. Rev., 2008, 37, 1783-1791.

10 Y. N. Xia, Y. J. Xiong, B. Lim and S. E. Skrabalak, Angew. Chem., Int. Ed., 2009, 48, 60-103.

11 N. M. Bedford, H. Ramezani-Dakhel, J. M. Slocik, B. D. Briggs, Y. Ren, A. I. Frenkel, V. Petkov, H. Heinz, R. R. Naik and M. R. Knecht, ACS Nano, 2015, 9, 50825092.

12 H. Ramezani-Dakhel, L. Ruan, Y. Huang and H. Heinz, Adv. Funct. Mater., 2015, 25, 1374-1384.

13 Z. Wang, G. Yang, Z. Zhang, M. Jin and Y. Yin, ACS Nano, 2016, 10, 4559-4564.

14 H. Zhang, M. Jin and Y. Xia, Angew. Chem., Int. Ed., 2012, 51, 7656-7673.

15 X. Q. Huang, Z. P. Zhao, J. M. Fan, Y. M. Tan and N. F. Zheng, J. Am. Chem. Soc., 2011, 133, 4718-4721.

16 M. T. M. Koper, Nanoscale, 2011, 3, 2054-2073.

17 B. A. Lu, J. H. Du, T. Sheng, N. Tian, J. Xiao, L. Liu, B. B. Xu, Z. Y. Zhou and S. G. Sun, Nanoscale, 2016, 8, 11559-11564.

18 N. Tian, Z.-Y. Zhou, S.-G. Sun, Y. Ding and Z. L. Wang, Science, 2007, 316, 732-735.

19 H. Jing, Q. Zhang, N. Large, C. Yu, D. A. Blom, P. Nordlander and H. Wang, Nano Lett., 2014, 14, 36743682.

20 M. Chen, B. Wu, J. Yang and N. Zheng, Adv. Mater., 2012, 24, 862-879.

21 B. Y. Xia, H. B. Wu, X. Wang and X. W. Lou, Angew. Chem., Int. Ed., 2013, 52, 12337-12340.

22 G. Chen, Y. Tan, B. Wu, G. Fu and N. Zheng, Chem. Commun., 2012, 48, 2758-2760.

23 X. Xia, J. Zeng, B. McDearmon, Y. Zheng, Q. Li and Y. Xia, Angew. Chem., Int. Ed., 2011, 50, 12542-12546.
24 H. Zhang, W. Li, M. Jin, J. Zeng, T. Yu, D. Yang and Y. Xia, Nano Lett., 2011, 11, 898-903.

25 M. J. Mulvihill, X. Y. Ling, J. Henzie and P. Yang, J. Am. Chem. Soc., 2010, 132, 268-274.

26 O. M. Magnussen, Chem. Rev., 2002, 102, 679-725.

27 S. E. Lohse, N. D. Burrows, L. Scarabelli, L. M. Liz-Marzán and C. J. Murphy, Chem. Mater., 2013, 26, 34-43.

28 S. Gómez-Grana, B. Goris, T. Altantzis, C. Fernández-López, E. Carbó-Argibay, A. Guerrero-Martínez, N. Almora-Barrios, N. López, I. Pastoriza-Santos, J. Pérez-Juste, S. Bals, G. Van Tendeloo and L. M. Liz-Marzán, J. Phys. Chem. Lett., 2013, 4, 2209-2216.

29 M. Liu, Y. Zheng, L. Zhang, L. Guo and Y. Xia, J. Am. Chem. Soc., 2013, 135, 11752-11755.

30 G. Wulff, Z. Krystallogr. Mineral., 1901, 34, 449-530.

31 R. Tran, Z. Xu, B. Radhakrishnan, D. Winston, W. Sun, K. A. Persson and S. P. Ong, Sci. Data, 2016, 3, 160080.

32 G. D. Barmparis, Z. Łodziana, N. López and I. N. Remediakis, Beilstein J. Nanotechnol., 2015, 6, 361-368.

33 K. Honkala, A. Hellman, I. N. Remediakis, A. Logadottir, A. Carlsson, S. Dahl, C. H. Christensen and J. K. Nørskov, Science, 2005, 307, 555-558.

34 K. Reuter and M. Scheffler, Phys. Rev. B: Condens. Matter, 2002, 65, 035406.

35 N. Almora-Barrios, G. Novell-Leruth, P. Whiting, L. M. LizMarzán and N. López, Nano Lett., 2014, 14, 871-875.

36 G. Kresse and J. Furthmüller, Phys. Rev. B: Condens. Matter, 1996, 54, 11169-11186.

37 G. Kresse and J. Furthmüller, Comput. Mater. Sci., 1996, 6, 15-50.

38 J. P. Perdew, K. Burke and M. Ernzerhof, Phys. Rev. Lett., 1996, 77, 3865-3868.

39 G. Kresse and D. Joubert, Phys. Rev. B: Condens. Matter, 1999, 59, 1758-1775.

40 P. E. Blochl, Phys. Rev. B: Condens. Matter, 1994, 50, 1795317979.

41 S. Grimme, J. Comput. Chem., 2006, 27, 1787-1799.

42 N. Almora-Barrios, G. Carchini, P. Błoński and N. López, J. Chem. Theory Comput., 2014, 10, 5002-5009.

43 J. Neugebauer and M. Scheffler, Phys. Rev. B: Condens. Matter, 1992, 46, 16067-16080.

44 M. Garcia-Ratés and N. López, J. Chem. Theory Comput., 2016, 12, 1331-1341.

45 M. Álvarez-Moreno, C. de Graaf, N. López, F. Maseras, J. M. Poblet and C. Bo, J. Chem. Inf. Model., 2015, 55, 95-103, https://iochem-bd.bsc.es/browse/handle/100/9.

46 N. E. Singh-Miller and N. Marzari, Phys. Rev. B: Condens. Matter, 2009, 80, 235407.

47 I. Chorkendorff and J. W. Niemantsverdriet, Concepts of Modern Catalysis and Kinetics, Wiley-VCH Verlag GmbH \& Co. KGaA, Weinheim, 2005.

48 H. Heinz and H. Ramezani-Dakhel, Chem. Soc. Rev., 2016, 45, 412-448. 